\title{
Preferred Suppliers in Auction Markets
}

\author{
Roberto Burguet \\ Institute for Economic Analysis (CSIC) \\ Campus UAB \\ Bellaterra, Barcelona, Spain 08193 \\ Martin K. Perry ${ }^{*}$ \\ Department of Economics \\ Rutgers University \\ New Brunswick, New Jersey, USA 08901
}

July 2008

\begin{abstract}
In a procurement setting, this paper examines agreements between a buyer and one of the suppliers which would increase their joint surplus. The provisions of such agreements depend on the buyer's ability to design the rules of the final procurement auction. When the buyer has no such ability, their joint surplus can be increased by an agreement which grants to the preferred supplier a right-of-first-refusal on the lowest price offer from the other suppliers. When the buyer does have this ability, one agreement which maximizes their joint surplus includes a revelation game for the cost of the preferred supplier and a reserve price in the procurement auction based on that cost.
\end{abstract}

Keywords: procurement auctions, bilateral agreements

JEL Classification: D44, D82, C79

\footnotetext{
* Martin would like to acknowledge the financial support of the Instituto de Analisis Economico (IAE, Institute for Economic Analysis) and the Institucio Catalana de Recerca i Estudis Avancats (ICREA, Catalan Institute for Research and Advanced Studies) in Barcelona Spain. Roberto would like to acknowledge financial support of FEDER and the Spanish Ministry of Science and Technology, projects SEC 2002-02506, SEJ2005-01427, and CREA. In addition, we would like to acknowledge Maria Lauve and Ying Zhang, Martin's research assistants at Rutgers University, for their help on various aspects of this paper. We would also like to thank Richard McLean, Martin's colleague at Rutgers University, for his careful suggestions on several theoretical issues. Finally this paper has greatly benefited from the comments by referees.
} 


\section{Introduction}

We investigate bilateral negotiations and the resulting agreements between a buyer and one potential supplier in a competitive procurement setting with multiple suppliers. In particular, we identify the existence of mutually beneficial agreements in which the buyer and one supplier affect the rules for future exchange.

In their influential paper entitled "Auctions versus Negotiations", Bulow and Klemperer (1996) show that auctions dominate negotiations. ${ }^{1}$ This result appears to contradict the findings of Aghion and Bolton (1987) in which a buyer and a supplier can specify a mutually beneficial agreement that would allow them to extract surplus from future potential suppliers. However, the apparent contradiction can be explained by the difference in the concept of negotiations employed in each paper. In Bulow and Klemperer, the term negotiations refers to any trading mechanism designed by the buyer in order to maximize his surplus from awarding a contract to suppliers with private information. In Aghion and Bolton, the term negotiations refers to a bilateral agreement between the buyer and one supplier in order to maximize their joint surplus. The agreement is reached at an ex ante stage prior to the realization of private information.

In this paper, we follow Aghion and Bolton by examining agreements which maximize the joint surplus of the buyer and one supplier, called the preferred supplier. As in Aghion and Bolton, these negotiations and the resulting agreement occur at the first stage, called stage 1, when there is no private information. In particular, the preferred supplier does not know her cost at stage 1, and thus has no informational advantage over the buyer during the negotiations. In the next stage, called stage 2, the preferred supplier and the other potential suppliers privately learn their costs. In the final procurement stage, called stage 3, the buyer awards the contract to one of the suppliers.

Under these assumptions, negotiations take place under symmetric information at stage 1 , and therefore any agreement should be Pareto-efficient and individually-rational. Moreover, all Pareto-efficient agreements will result in the same trades at stage 3, and will only differ in the transfers between the buyer and the preferred supplier at stage 1. By assuming no budget or financial constraints, our model does not attempt to identify which of the possible transfers will take place. 
We first examine agreements at stage 1 between the buyer and the preferred supplier in which the buyer grants to the preferred supplier a right-of-first-refusal that can be exercised at stage 3. ${ }^{2}$ With a right-of-first-refusal, hereafter denoted ROFR, the preferred supplier has the option to be awarded the contract at a price equal to the lowest offer that the buyer obtains from the other competing suppliers.

Bikhchandani, Lippman, and Ryan (2005) showed that a ROFR cannot increase the joint surplus of a buyer and a preferred supplier when the buyer uses a second-price auction. However, a second-price auction is not an appropriate model for a market when the buyer has no ability to design and commit to the rules of trade in stage 3. This is the first case that we consider. In particular, we examine markets in which the buyers do not have this ability. The suppliers make price offers to the buyer who then simply decides which offer to accept. If suppliers make (simultaneous) take-it-or-leave-it price offers to the buyer, such a market would be naturally characterized as a first-price auction. For two suppliers, Choi (forthcoming) has shown that when suppliers make price bids (that is, when trade is governed by a first-price auction), the buyer and the preferred supplier can gain from a ROFR. The analysis of Choi does not extend to more than two potential suppliers. However, under some assumptions on the distribution of costs, we show in section 3 that Choi's findings can be generalized to any number of potential suppliers, and that a $R O F R$ can increase the joint surplus of the buyer and one preferred supplier.

A second-price auction is also not an appropriate model for a market where suppliers can improve their offers in response to the offers of other suppliers. Indeed, when no other remaining suppliers are willing to improve on the last offer by one supplier, that supplier may still make a final offer reducing his price in order to prevent the contract from being awarded to the preferred supplier who has a $R O F R .^{3}$ Thus, such a market is better modeled as an oral auction where the "winner" is able to make a final offer to the buyer that improves on his last offer. Using this

\footnotetext{
${ }^{1}$ In the context of procurement, the authors have shown was that a simple auction would result in a lower expected price than an "an optimally-structured negotiation with one less bidder."

${ }^{2}$ These preferential treatments are usually the product of agreements in the past, many times as by-product of other transactions. That is, they are the result of negotiations carried out at a time where the informational asymmetry with respect to the present transaction was less important.

${ }^{3}$ This is impossible under the rules of a second-price auction. Although this difference is irrelevant when no supplier has a $R O F R$, it happens to be crucial when there is a supplier with a ROFR. Thus, the results in Bikhchandani, Lippman, and Ryan (2005) hold when suppliers participate in a second-price auction, a designed mechanism when bidders do not make price offers, rather than a decentralized market where suppliers decide on the prices they offer.
} 
modified oral auction, we show in section 3 that a $R O F R$ increases the joint surplus of the buyer and the preferred supplier for any distribution of costs.

The $R O F R$ satisfies two natural constraints on the feasible agreements at stage 1 when the buyer does not have the ability to commit to the rules of trade in stage 3. Given the price offers of competing suppliers at stage 3, a ROFR does not require the buyer to pay a price higher than the lowest price offer received from the suppliers. Also, the ROFR does not require the preferred supplier to accept the contract at a price below her cost. ${ }^{4,5}$ In addition, the ROFR maximizes the ex post gains from trade in stage 3 between the buyer and the preferred supplier. Thus, the ROFR is a natural provision to consider for an agreement in stage 1 when the buyer does not have the ability to commit to the rules of trade in stage 3 . However, more complex provisions for this agreement may arise when the buyer does have this ability. In section 4, we examine an optimal agreement when the buyer can commit to the rules of trade in stage 3.

In general, the mechanism that would maximize the buyer's ex ante surplus would not specify trading rules that would maximize the ex post gains from trade in stage 3 . These optimal trading rules often create ex post inefficiencies in order to extract surplus from one or more suppliers. Without an agreement in stage 1, the optimal mechanism for the buyer would specify a standard auction with a reserve price below the buyer's value. As such, the buyer creates a positive probability of refusing to award the contract even though some supplier would be willing to make a price offer below his value, though not below his reserve price. ${ }^{6}$ Standard auction theory implies that the mechanism which maximizes the expected joint surplus of the buyer and the preferred supplier would be a standard auction with an even lower reserve price that depends on the reported cost of the preferred supplier. However, in order to implement this mechanism, the

\footnotetext{
${ }^{4}$ Of course, one can envision an agreement in which the preferred supplier is required to supply the good for a set price at the option of the buyer. Such an agreement could create a reserve price at the procurement stage below both the reservation value of the buyer and the cost of the preferred supplier. This is a trivial way of recreating commitment ability when we are trying to examine the ROFR in the absence of such ability.

${ }^{5}$ Our results on the ROFR can be extended very easily to show that granting the ROFR to one supplier increases the joint surplus of the buyer and the preferred supplier even when the buyer uses an optimal auction to maximize his surplus, such as when the buyer can set a binding reserve price. However, if the buyer can design the trading rules, that agreement does not maximize the joint surplus of the buyer and the preferred supplier. Arozamena and Weinschelbaum (2006) show that a ROFR cannot be part of the optimal mechanism for the buyer in this case.

${ }^{6}$ Hua (2007) considers this case reversing the role of buyers and sellers (a seller faces multiple buyers). He finds the optimal contract when the preferred buyer has limited funds at stage 1 . When the preferred buyer has sufficient funds at stage 1 , as in our case, that contract amounts to selling the object at stage 1 to the preferred buyer, who then can auction it to other buyers. Or, in our procurement setting, this agreement includes payments to the preferred supplier that depend on the cost of competing suppliers. This may be problematic if we are discussing agreements other than full vertical integration between the buyer and the preferred supplier.
} 
buyer would need to learn the cost of the preferred supplier that is privately realized at stage 2. In section 4 we demonstrate that this mechanism can indeed be implemented by an agreement in stage 1 which incorporates a revelation game in stage 2.

\section{The procurement model}

We examine a three-stage procurement model with one buyer and multiple suppliers each having independent private costs. The buyer has a value $(v>1)$ for a good or service having a fixed quantity and quality. There are $(n+1)$ suppliers, and each supplier obtains its cost of production $c_{i}$ as an independent realization of a random variable with the distribution function $G(c)$ on the support [0,1]. For some specific results, we will also need additional assumptions on the cost distribution. First, we may need to assume that the density function $g(c)$ is continuously differentiable. Second, we may need to assume that the inverse hazard rate function, defined as $[1-G(c)] / g(c)$, is monotonically decreasing in $c$. Third, we may also need to assume that $G(c) / g(c)$ is increasing in $c$. These assumptions on the cost distribution are common in procurement auction models.

The procurement model has three stages. At the beginning of stage 1 , the value of the buyer $v$ and the cost distribution $G(c)$ of the suppliers are common knowledge. During stage 1, the buyer can negotiate an agreement with one preferred supplier (PS) about the rules and payoffs for the future stages in the procurement process. At the beginning of stage 2, each supplier realizes his cost of production $c_{i}$, and the cost $c_{i}$ is private information to the $i$ th supplier. At the end of stage 3, the buyer awards the contract for the good to one of the suppliers and pays that supplier.

In section 3, we examine two auction models for stage 3, a standard first-price auction and a modified open auction. With the first-price auction, suppliers can make simultaneous price offers to the buyer at stage 3. If there is no agreement at stage 1, then the buyer accepts the lowest offer. We assume that the accepted lowest offer can be verified. However, if there is an agreement at stage 1 to grant a $R O F R$, the PS has the right to accept the contract at the lowest price offer from the other suppliers. If the PS chooses not to accept the contract at that price, the buyer awards the contract to the supplier with the lowest offer. We assume that the presence of the PS with the ROFR is common knowledge. 
In section 3, we also examine a modified open auction, modeled as a clock auction, in which suppliers make sequentially lower price offers at stage 3 until all but one supplier drops out. At that point, the remaining supplier can make a final reduction in his price offer. If there is no agreement at stage 1 , this supplier obtains the contract at that price. If there is an agreement at stage 1, the PS can again exercise the ROFR to accept the contract at that same price.

In section 4, we examine the case in which the buyer can design the trading mechanism used at stage 3. If there is no agreement at stage 1 , the buyer designs the mechanism that maximizes his surplus. If there are negotiations at stage 1 , the agreement between the buyer and the PS can set the trading rules in stage 3.

For the models in both sections 3 and 4, the buyer and the PS have symmetric information at stage 1. Thus, any agreement between them would result in an ex ante Paretoefficient outcome, that is, one that maximizes their expected joint surplus. The resulting division of this joint surplus between the buyer and the PS would be implemented by transfers between the buyer and the PS at stage 1. These transfers do not affect the allocation of the contract.

\section{Agreement with a right-of-first-refusal}

In this section, we examine potential agreements between the buyer and a PS in which the buyer grants a ROFR to the PS. First, we consider the case of a first-price auction in stage 3. Without an agreement at stage 1 , we define the outcome of that first-price auction. ${ }^{7}$ All $(n+1)$ suppliers make simultaneous price offers, and the buyer awards the contract to the supplier with the lowest offer at a price equal to that offer. The expected profit of a supplier after its cost is known at stage 2 can be expressed as

$$
\Pi(c)=\int_{c}^{1}[1-G(z)]^{n} d z .
$$

The resulting expected profits of a supplier at stage 1 are

$$
E \Pi=\int_{0}^{1} G(c) \cdot[1-G(c)]^{n} d c .
$$

With symmetric suppliers, the expected price paid by the buyer with this first-price auction is

$$
E P_{F P A}=\int_{0}^{1} c \cdot(n+1) \cdot n \cdot g(c) \cdot G(c) \cdot[1-G(c)]^{n-1} d c .
$$


Now assume that the buyer and the PS negotiate an agreement that grants a $R O F R$ to the PS, perhaps in exchange for some transfer by the PS to the buyer during stage 1 . We use the term preference auction (PA) to define the first-price auction in which one supplier has a ROFR, and denote the other $n$ suppliers as the competing suppliers (CS). After receiving the price offers from the CS, the buyer then offers the contract to the PS at the lowest price offered by the CS. The PS will accept contract if her cost is below the lowest offer of the CS, and will reject it otherwise. $^{8}$ If the contract is rejected by the PS, the buyer then awards the contract to the CS with the lowest offer at a price equal to that offer.

We will subscript variables related to the preferred supplier (PS) by $p$ and those related to the competing suppliers (CS) by $k$. We now characterize a symmetric, monotone equilibrium bidding function for the CS, $b(c)$. This equilibrium bidding function must satisfy:

$$
c=\arg \max _{z} \Pi_{k}[b(z) ; c]=\arg _{\max _{z}}(b(z)-c) \cdot[1-G(b(z))] \cdot[1-G(z)]^{n-1} .
$$

In order for a particular CS to win the contract, its bid $b(z)$ must be below the cost of the PS and its "pretended" cost $z$ must be below the costs of each of the other $(n-1)$ CS. When $n=1$, the maximization problem in (4) is dominance solvable. However when $n>1$, the first-order condition for this problem is the following differential equation, ${ }^{9}$

$$
b^{\prime}(\mathrm{c})=\frac{H(b) \cdot(n-1) \cdot[b(c)-c]}{H(c) \cdot\{H(b)-[b(c)-c]\}}
$$

where $H$ represents the inverse hazard rate $H(x)=[1-G(x)] / g(x)$. Together with the condition $b(1)=1$, the differential equation (5) is an initial value problem whose solution defines the equilibrium, symmetric bidding function $b(c)$ for CS when $n>1 .{ }^{10}$ Although standard existence

\footnotetext{
${ }^{7}$ The derivation of these results is standard. See Myerson (1981).

${ }^{8}$ The PS has no incentive to make an offer because any offer below all the offers of the CS would only lower the price at which the PS would be awarded the contract. See Burguet and Perry (2007) for an alternative model of favoritism.

${ }^{9}$ Monotonicity and symmetry of the bounded bidding function imply that it is differentiable almost everywhere.

10 The second-order conditions are satisfied as long as $b(c)>c$ at the solution to the differential equation (5), and then $b^{\prime}(c)>0$. Indeed, the first-order condition defines an identity in $c,\left.\frac{\partial \Pi_{k}[b(z) ; c]}{\partial z}\right|_{z=c}=0$, so that
} 
and uniqueness results do not apply, we can still prove the existence and uniqueness of this bidding function under certain assumptions on the cost distribution $G(c)$.

Proposition 1: If the density function $g(c)$ is continuously differentiable, then there exists a unique strictly increasing equilibrium bidding function $b(c)$ for the competing suppliers in a preference auction defined by (5) and $b(1)=1$.

The proof of Proposition 1 contained in the Appendix. We can now calculate the expected profits of the suppliers and the expected price for the buyer at stage 1 . In order to do so, we first calculate the expected profit function of both the PS and the CS in stage 2 after they have realized their cost $c$. In a monotone equilibrium, incentive compatibility requires that the derivative of the expected profit function of a CS is $\partial \Pi_{k}(c) / \partial c=-[1-G(b(c))] \cdot[1-G(c)]^{n-1}$. By integrating this expression, we obtain the expected profit function of a CS with cost $c$ :

$$
\Pi_{k}(c)=\int_{c}^{1}[1-G(b(z))] \cdot[1-G(z)]^{n-1} d z .
$$

Similarly, we obtain the expected profit function of the PS with cost $c$ :

$$
\Pi_{p}(c)=\left\{\begin{array}{ll}
{[b(0)-c]+\int_{b(0)}^{1}\left[1-G\left(b^{-1}(z)\right)\right]^{n} d z} & \text { if } c \leq b(0) \\
\int_{c}^{1}\left[1-G\left(b^{-1}(z)\right)\right]^{n} d z & \text { if } b(0)<c<1
\end{array} .\right.
$$

From these expressions, we can calculate the expected profits of each type of supplier over the distribution of costs:

$$
\begin{aligned}
& E \Pi_{k}=\int_{0}^{1} G(c) \cdot[1-G(b(c))] \cdot[1-G(c)]^{n-1} d c \\
& E \Pi_{p}=\int_{b(0)}^{1} G(c) \cdot\left[1-G\left(b^{-1}(c)\right)\right]^{n} d c+\int_{0}^{b(0)} G(c) d c .
\end{aligned}
$$

$$
\begin{aligned}
& \left.\frac{d}{d c} \frac{\partial \Pi_{k}[b(z) ; c]}{\partial z}\right|_{z=c}=\left.\frac{\partial}{\partial z} \frac{\partial \Pi_{k}[b(z) ; c]}{\partial z}\right|_{z=c}+\left.\frac{\partial}{\partial c} \frac{\partial \Pi_{k}[b(z) ; c]}{\partial z}\right|_{z=c}=0 \text {. Thus the second-order condition is } \\
& \text { simply }\left.\frac{\partial}{\partial c} \frac{\partial \Pi_{k}[b(z) ; c]}{\partial z}\right|_{z=c}=-\frac{d[1-G(b(c))][1-G(c)]^{n-1}}{d c} \geq 0 .
\end{aligned}
$$


Expressions (8) and (9) are the expected profits for each type of supplier at the end of the stage 1, after the buyer and the PS have negotiated the agreement for a $R O F R$, but before any supplier has realized its cost in stage 2. From (2), (8), and (9), it follows immediately that

$$
E \Pi_{p}>E \Pi>E \Pi_{k} \text {. }
$$

Relative to a first-price auction without preference for any supplier, the PS benefits from the $R O F R$ in the preference auction, while each CS is harmed. Finally, the expected price for the buyer in stage 1 from the preference auction in stage 3 is the expected value of the lowest price offered by the CS:

$$
E P_{P A}=\int_{0}^{1} b(c) \cdot n \cdot g(c) \cdot[1-G(c)]^{n-1} d c
$$

We now compare the joint surplus of the buyer and the PS with and without the ROFR. Since the probability that the buyer will award the contract to one of the suppliers is the same with or without preference, we need only examine the difference between the ex ante expected profits of the PS in stage 1 and the ex ante expected prices for the buyer in stage 1. We compare this difference with and without the ROFR. Under certain assumptions on inverse hazard rate function, the ROFR will indeed increase the expected joint surplus of the buyer and the PS.

Proposition 2: If the inverse hazard rate function $[1-G(c)] / g(c)$ is decreasing and convex, then the expected joint surplus of the buyer and the preferred supplier is higher when the buyer grants a right-of-first-refusal to the preferred supplier.

Proof of Proposition 2: The difference between the joint surplus with and without preference is $\left[E \Pi_{p}+\left(v-E P_{P A}\right)\right]-\left[E \Pi+\left(v-E P_{F P A}\right)\right]$. Rearranging this difference in terms of the profits and prices yields $\left[E P_{F P A}-E P_{P A}\right]+\left[E \Pi_{p}-E \Pi\right]$. Denote $b_{F P A}(c)$ as the bidding function in a first-price auction with $(n+1)$ symmetric suppliers. The expected price in this first-price auction without preference can be decomposed as follows: 


$$
\begin{aligned}
E P_{F P A}= & \int_{0}^{1} b_{F P A}(c) \cdot(n+1) \cdot g(c) \cdot[1-G(c)]^{n} d c \\
= & \int_{0}^{1} b_{F P A}(c) \cdot n \cdot g(c) \cdot[1-G(c)]^{n-1} d c-\int_{0}^{1} b_{F P A}(c) \cdot n \cdot g(c) \cdot G(c) \cdot[1-G(c)]^{n-1} d c \\
& \quad+\int_{0}^{1} b_{F P A}(c) \cdot g(c) \cdot[1-G(c)]^{n} d c .
\end{aligned}
$$

The expected profits in the preference auction can be expressed directly in terms of the bidding function, and then can be bounded from below as follows:

$$
\begin{aligned}
E \Pi_{p} & =\int_{0}^{1}\left(\int_{\max \left\{b^{-1}(c), 0\right\}}^{1}(b(x)-c) \cdot n \cdot g(x) \cdot[1-G(x)]^{n-1} d x\right) g(c) d c \\
& >\int_{0}^{1}\left(\int_{c}^{1}(b(x)-c) \cdot n \cdot g(x) \cdot[1-G(x)]^{n-1} d x\right) g(c) d c \\
& =\int_{0}^{1} \int_{c}^{1} b(x) \cdot n \cdot g(x) \cdot[1-G(x)]^{n-1} d x \cdot g(c) d c-\int_{0}^{1} c \cdot g(c) \cdot[1-G(c)]^{n} d c \\
& =\int_{0}^{1} b(c) \cdot n \cdot g(c) \cdot[1-G(c)]^{n-1} \cdot G(c) d c-\int_{0}^{1} c \cdot g(c) \cdot[1-G(c)]^{n} d c .
\end{aligned}
$$

The inequality in the second line arises from the fact that $c>\max \left\{b^{-1}(c), 0\right\}$, and the equality in the last line is obtained by changing the order of integration. After substituting these two expressions, and the expression for the $E P_{P A}$ from (11), into the difference in joint surplus, and rearranging terms, we find that

$$
\begin{gathered}
{\left[E P_{F P A}-E P_{P A}\right]+\left[E \Pi_{p}-E \Pi\right]>\int_{0}^{1}\left[b_{F P A}(c)-b(c)\right] \cdot n \cdot g(c) \cdot[1-G(c)]^{n-1} d c} \\
-\int_{0}^{1}\left[b_{F P A}(c)-b(c)\right] \cdot n \cdot g(c) \cdot G(c) \cdot[1-G(c)]^{n-1} d c \\
+\left(\int_{0}^{1}\left[b_{F P A}(c)-c\right] \cdot g(c) \cdot[1-G(c)]^{n} d c-E \Pi\right) .
\end{gathered}
$$

The third term on the right-hand side is zero because the integral is the definition of the expected profits $E \Pi$ of a supplier in the auction without preference. Combining the first two terms, we obtain the following lower bound on the difference in the joint surplus:

$$
\left[E P_{F P A}-E P_{P A}\right]+\left[E \Pi_{p}-E \Pi\right]>\int_{0}^{1}\left[b_{F P A}(c)-b(c)\right] \cdot n \cdot g(c) \cdot[1-G(c)]^{n} d c
$$

Arozamena and Weinschelbaum (2004) and Porter and Shoham (2005) have examined the bidding behavior of $n$ symmetric suppliers in a first-price auction when another supplier has a 
ROFR. Both papers demonstrate that $\left[b_{F P A}(c)-b(c)\right]$ is positive for all $c$ if the inverse hazard rate function of the cost distribution $[1-G(c)] / g(c)$ is decreasing and convex. QED

Proposition 2 provides insight into how the ROFR extracts rents from the CS. If the inverse hazard rate function is decreasing and convex, then the CS make more aggressive price offers after the ROFR is granted to one supplier. Expected total surplus of the buyer and all the suppliers is reduced because the ex post allocation in the preference auction will be inefficient for certain cost realizations. In particular, an inefficient allocation occurs when the cost of the PS is below the lowest offer of the CS, but above the cost of the CS with the lowest offer. However, Proposition 2 demonstrates that the expected value of this inefficiency is smaller than the reduction in expected surplus of the CS. As a result, the expected joint surplus of the buyer and the PS increases with an agreement in stage 1 whereby the buyer grants a ROFR to the PS. This generalizes the results in Choi (forthcoming) for any number of CS.

Proposition 2 also demonstrates that the findings of Bikhchandani, Lippman, and Ryan (2005) are unique to a second-price auction among the CS. Once the ROFR is granted to one supplier, a second-price auction among the CS is no longer equivalent to a first-price preference auction among the CS. With a second-price auction, the dominant strategy for a supplier is to make a price offer equal to his cost with or without preference. As such, the CS would not bid more or less aggressively in the presence of a PS. This is not true in the case of a first-price auction. Depending on the properties of the inverse hazard rate function, the CS may bid more or less aggressively in the presence of a PS.

Now, assume that the buyer can "shop" for lower prices. Absent agreements at stage 1, the market is best described as an oral auction. We examine a modified open auction which takes the form of a clock auction, ${ }^{11}$ with a final offer by the winner. The clock points to progressively lower prices until all but one supplier drop out of the auction. At this moment, the one supplier that has not dropped out can make this price his final offer, or can make an even lower offer. Without an agreement between the buyer and the PS, the winner never has an incentive to make a lower final offer. Thus, this auction is equivalent to a standard clock auction, and has a simple (perfect-Bayesian) equilibrium where all suppliers drop out at their cost, and the winner makes the price on the clock his final offer. Thus, without an agreement at stage 1, equations (2) and (3) 
still describe the payoffs of all agents. If there is an agreement, Proposition 3 describes an equilibrium at stage 3 .

Proposition 3: In the modified open auction where the preferred supplier has a right-offirst-refusal the following describes the equilibrium strategy for each competing supplier with cost $c_{i}: \mathrm{i}$ ) if not all other competing suppliers have dropped out, drop out at a price equal to $c_{i}$; ii) if all other competing suppliers have dropped out, and the last of them dropped out at price $p$, then make a final offer equal to $\min \left\{p, p^{\prime}\left(c_{i}\right)\right\}$, where $p^{\prime}\left(c_{i}\right)$ is the solution to

$$
p^{\prime}-\frac{1-G\left(p^{\prime}\right)}{g\left(p^{\prime}\right)}=c_{i} .
$$

For the preferred supplier, her equilibrium strategy is to accept the contract if this final offer is below her cost.

The proof of Proposition 3 is straightforward, and we omit it. As in a second-price auction, the equilibrium price will coincide with the second lowest cost realization of the CS when this second lowest cost is close to the lowest cost realization of the CS. However, if the lowest cost is sufficiently below the second lowest cost, then the equilibrium price defined by (13) will be lower than it would in a second-price auction. This observation is almost all that is necessary to conclude that a $R O F R$ results in higher expected joint surplus for the buyer and the PS.

Proposition 4: The expected joint surplus of the buyer and the preferred supplier is higher when the buyer grants to the preferred supplier a right-of-first-refusal.

Proof of Proposition 4: Let $c_{1}$ and $c_{2}$ represent the lowest and second lowest cost realization of CS, respectively. Also, let $p^{\prime}\left(c_{1}\right)$ be the solution of (13) at $c_{1}$. If $p^{\prime}\left(c_{1}\right)>c_{2}$, then the price will be $c_{2}$ with or without a ROFR. The profit of the PS will also be the same and there could be no difference in the joint surplus. Now, suppose that $p^{\prime}\left(c_{1}\right)<c_{2}$. If so, the price $p^{\prime}\left(c_{1}\right)$ with the ROFR will be less than the price $c_{2}$ without the ROFR. Now consider the joint surplus

\footnotetext{
${ }^{11}$ See, Cramton (1998) for a description of this model.
} 
of the buyer and the PS when $c_{p}$ is the cost of the PS. If $c_{p}<p^{\prime}\left(c_{1}\right)$, then the contract is awarded to the PS with or without the ROFR. Thus, again there would be no difference in the joint surplus, $v-c_{p}$. If $c_{p}>p^{\prime}\left(c_{1}\right)$, then the contract is awarded to the lowest cost CS at a price $p^{\prime}\left(c_{1}\right)$ with the ROFR. The resulting joint surplus will be $v-p^{\prime}\left(c_{1}\right)$. Without the $R O F R$, the joint surplus will be $v-c_{2}$ when $c_{2} \leq c_{p}$, and $v-c_{p}$ when $c_{2}>c_{p}$. Since $p^{\prime}\left(c_{1}\right)<\min \left\{c_{2}, c_{p}\right\}$, the joint surplus with the ROFR is greater than the joint surplus without the ROFR. QED

One might ask whether there could be another agreement that would result in even higher joint surplus. For the moment assume that CS must make their price offers, final or not, before the agreement in stage 1 is implemented, and that the buyer and the PS take these offers as given, independent of their agreement. Under these conditions, ex ante maximization of joint surplus is equivalent to ex post maximization of the gains from trade, and a ROFR accomplishes both. Although the $R O F R$ requires that the lowest price offer from CS is verifiable, ${ }^{12}$ the $R O F R$ is immune to problems associated with asymmetric information between the buyer and the PS on the cost of the PS.

Thus, the answer to this question depends on whether an agreement at stage 1 can influence the price offers by the CS. Whatever the agreement states, that will not occur if the buyer trades with CS if and only if their lowest offer is below the cost of the PS. Indeed, the CS will solve the same problem whose solution is characterized in Proposition 1 or Proposition 3. We are assuming that the buyer cannot commit to trade at a price above the lowest price offer he receives and that the PS cannot commit to trade at a price below her cost, Thus, the only remaining possibility is that, as a consequence of the agreement at stage 1 , there is a positive probability that the buyer trades with a CS at a price above the cost of the PS. Although it seems counterintuitive that more aggressive price offers by the CS could be induced (and so more surplus could be extracted from CS) by increasing the probability that they obtain the contract for a given price, in fact we cannot rule out this possibility in general. Thus, in principle, a ROFR may not be a provision of the optimal agreement when the buyer cannot commit to the rules of trade. However, it also seems theoretically challenging, at best, and certainly beyond the

\footnotetext{
${ }^{12}$ Verifiability of this offer (the realized price, if trade takes place with a CS) is a relatively weak condition because the ROFR requires that such price be communicated to the PS.
} 
goals of this paper, to characterize the set of implementable allocations that also satisfy the notion of "inability to commit" on the part of the buyer.

\section{Mechanism design and bilateral negotiations.}

In this section, we examine agreements in stage 1 when the buyer has the ability to commit to the rules of trade to be used in stage 3. In particular, we characterize the optimal agreement between the buyer and the PS under this assumption.

Let $c_{p}$ denote the cost realization of the PS in stage 2. The buyer and the PS could guarantee themselves a minimum joint surplus of $\left(v-c_{p}\right)$ at stage 2 by simply awarding the contract to the PS. Thus, if there were no informational asymmetries at stage 2 , the cost $c_{p}$ of the PS becomes the joint willingness to pay by the buyer and PS for awarding the contract to one of the CS. Standard auction theory would then apply to this case. For an agent whose willingness to pay is $x$, the optimal mechanism is a standard auction with reserve price $r(x)$ defined by:

$$
r+\frac{G(r)}{g(r)}=x .
$$

Thus, the mechanism that maximizes the expected joint surplus would be a standard auction with a reserve price $r\left(c_{p}\right)$. Given our assumption on the distribution function that $G(c) / g(c)$ is increasing in $c$, the left-hand side of (15) is clearly increasing in $r$. Thus, $r\left(c_{p}\right)$ is an increasing function of the cost of the PS.

This first-price auction with an optimal reserve price defined by (15) is not a feasible (implementable) agreement at stage 1. Unlike the previous agreement in which the buyer granted a $R O F R$ to the PS, an agreement to implement this auction requires that the buyer learn the cost of the PS before the procurement stage 3. Thus, the agreement must account for the incentives of the PS to reveal her cost to the buyer in stage 2. The question is whether an agreement between the buyer and the PS can be designed in stage 1 so that the PS has an incentive to reveal her cost at stage 2. Proposition 5 provides an affirmative answer to this question. 
Proposition 5: The following agreement between the buyer and the preferred supplier maximizes their expected joint surplus. At stage 2, the preferred supplier reports her cost $c_{p}$ to the buyer in exchange for a payment from the buyer equal to $\int_{c}^{1}[1-G(r(x))]^{n} d x$, where $r(x)$ is defined by (15). At stage 3, the buyer then holds a first-price auction among the competing suppliers with a optimal reserve price $r\left(c_{p}\right)$. If none of the competing suppliers submits a bid below $r\left(c_{p}\right)$, the buyer awards the contract to the preferred supplier at a price equal to $c_{p}$.

Proof of Proposition 5: We need to show that the payment from the buyer provides the PS with an incentive to reveal her true cost $c_{p}$ at stage 2. Consider the revelation problem facing the PS when deciding on her cost report to the buyer:

$$
\max _{z} \int_{z}^{1}[1-G(r(x))]^{n} d x+\left(z-c_{p}\right) \cdot[1-G(r(z))]^{n} .
$$

The first term is the payment for the cost report $z$. The second term is the expected profit (or loss) from a cost report $z$ above (or below) the actual cost of the PS, equal to the margin times the probability of being awarded the contract at price $z$. The first-order condition for this problem is

$$
-\left(z-c_{p}\right) \cdot n \cdot[1-G(r(z))]^{n-1} \cdot g(r(z)) \cdot r^{\prime}(z)=0 .
$$

This condition is satisfied at $z=c_{p}$. Using the same argument as footnote 15, the second-order condition is also satisfied at $z=c_{p}$. In particular, the derivative of the left-hand side of (17) with respect to $z$ equals the negative of its derivative with respect to $c_{p}$, $-n \cdot[1-G(r(z))]^{n-1} \cdot g(r(z)) \cdot r^{\prime}(z)<0$, which is negative. QED

In the absence of an agreement at stage 1, the buyer's optimal mechanism at stage 2 is a first-price auction with a reserve price $r(v)$. The mechanism of Proposition 5 induces higher expected joint surplus for the buyer and the PS. With respect to efficiency, the results are ambiguous. In a first-price auction with reserve price $r(v)$ and no $R O F R$, the allocation is efficient provided that trade occurs. This is not necessarily so with the mechanism of Proposition 5. Thus, for examples where $r(v)=1$, the mechanism is less efficient than the auction. However, 
trade always occurs in the mechanism of Proposition 5, and this implies a gain in efficiency with respect to any auction where $r(v)<1$. Thus, contrary to the cases analyzed in section 3 , when the buyer can commit to the trading rules, the agreement between the buyer and the PS has an ambiguous effect on efficiency.

As an illustration, assume that costs are distributed uniformly and that there are only two suppliers, $n=1$. If $v=2$, we find that $r(v)=1$, and $r\left(c_{p}\right)=1 / 2 c_{p}$. In this case the expected total surplus is larger with the auction than with the mechanism of Proposition 5. However, when $v=1$, we find that $r(v)=1 / 2$ and $r\left(c_{p}\right)$ is unchanged. In this case, the expected total surplus generated by the auction with the reserve price $r(v)$ is $7 / 12$, whereas the expected total surplus under the mechanism of Proposition 5 is 9/12. Thus, an agreement at stage 1 results in more efficient trade, i.e., increases the expected total surplus.

\section{Concluding Remarks}

There are two extreme cases which this paper does not (and cannot) address. The first case occurs when bilateral negotiations cannot be conducted prior to the realization of the costs. This would prohibit lump sum transfers (i.e., independent of the cost realizations) between the buyer and the preferred supplier. In other words, the (interim) individual rationality constraint for the preferred supplier would have to be satisfied for each cost realization. Myerson and Satterthwaite (1983) showed that the buyer and the seller could not realize the joint surplus that is attained with the mechanism of Proposition 5 (i.e., could not realize Pareto-optimal negotiations). Moreover, it is a relatively straightforward corollary of the analysis in Myerson (1981) that no bilateral negotiation could simultaneously allow the buyer and a PS to realize an expected joint surplus above what could be obtained from an auction with reserve price independent of the cost of the preferred supplier while inducing the supplier to reveal his cost, and allowing the buyer to appropriate a share of the joint surplus greater than what he could obtain alone using an auction with an appropriate reserve price.

The second case occurs when all parties can participate in the ex ante negotiations without restriction. In this case an auction (with a reserve price $v$, i.e., no effective reserve price)

clearly maximizes expected total surplus. However, even if all-inclusive negotiations are feasible, the possible outcomes of bilateral negotiations may be relevant for defining the threat 
points. Consider the case in which a buyer negotiates ex ante with two potential suppliers. For example, Burguet, Caminal, and Matutes (2002) have a model of three party negotiations in this setting. If one of the two suppliers departed the negotiations, the mechanism in Proposition 5 defines the fall-back option of the other two parties when they can design the trading mechanism. That is, the mechanism determines the highest expected joint surplus that they can guarantee themselves knowing that in the future they could contact the other supplier for trading. Moreover, the grant of a ROFR would determine the fall-back option when it is the departing supplier who would have the ability to set the terms of trade in this future potential contact.

The usual justification for bilateral negotiations, instead of all-inclusive negotiations, is that the mere existence or availability of these other competing suppliers is a random event. In other words, exogenous entry is possible in the future (altering our stage 1), but is not a certain event. See Aghion and Bolton (1987) or Hua (2007). It is a straightforward exercise to extend the analysis in this paper to such case. 


\section{References}

Aghion, Philippe and Patrick Bolton. "Contracts as a Barrier to Entry." American Economic Review, Vol. 77 (1987), pp. 388 -401.

Arozamena, Leandro and Federico Weinschelbaum. "The Effect of Corruption on Bidding Behavior in First-Price Auctions.” working paper, U. Torcuato di Tella and U. San Andrés, 2004.

Arozamena, Leandro and Federico Weinschelbaum. "A note on the suboptimality of right-of-first-refusal clauses." Economics Bulletin, Vol. 4(24) (2006) pp. 1-5.

Bikhchandani, Sushil, Steven Lippman, and Reade Ryan. "On the Right-of-FirstRefusal." Advances in Theoretical Economics, Vol. 5(1) (2005) Article 4.

Burguet, Roberto, Ramon Caminal, and Carmen Matutes. "Golden Cages for Showy Birds; Endogenous Switching Cost in Labor Contracts." European Economic Review, (journal of the European Economic Association) Vol. 46(7) (2002), pp. 1153-1185.

Burguet, Roberto and Martin K. Perry. "Bribery and Favoritism by Auctioneers in Sealed-Bid Auctions.” Contributions to Theoretical Economics, 2007.

Choi, Albert H. "A Rent Extraction Theory of Right of First Refusal," forthcoming in Journal of Industrial Economics.

Cramton, Peter. “Ascending Auctions,” European Economic Review Vol 42:3-5 (1998) pp. 745-756.

Hua, Xinyu. "Strategic Ex Ante Contracts: Rent Extraction and Opportunity Costs." RAND Journal of Economics, Vol. 38(3) (2007), pp. 786-803.

Myerson, Roger B. "Optimal Auction Design." Mathematics of Operations Research, Vol. 6 (1981), pp. 58-73.

Myerson, Roger B., and Mark Satterthwaite. "Efficient Mechanisms for Bilateral Trading.” Journal of Economic Theory, Vol. 29 (1983), pp. 265-28.

Porter, Ryan, and Yoav Shoham. "On Cheating in Sealed-Bid Auctions.” Decision Support Systems, Vol. 39(1) (2005), pp. 41-54. 


\section{Appendix: Proof of Proposition 1}

We need only prove that the initial value problem in (5) with $b(1)=1$ has a unique, strictly monotone solution whenever $g(c)$ is continuously differentiable. Write the initial value problem (5) as $b^{\prime}=\phi(b, c), b(1)=1$, where

$$
\phi(b, c)=\frac{H(b) \cdot(n-1) \cdot[b-c]}{H(c) \cdot\{H(b)-[b-c]\}}
$$

We cannot readily apply standard existence results to this initial value problem because $H(1)=0$ and then $\phi(b, c)$ is not well-defined around $(1,1)$. The problem occurs in the denominator of $\phi(b, c)$. Let us define $B(c)$ as the inverse function of $B^{-1}(c)=x-H(x)$. Note that the denominator in the definition of $\phi(b, c)$ is 0 at any point $(c, B(c))$. At any point $(c, b)$ with $b<B(c)$, the denominator is positive and $\phi(b, c)$ is well-defined. Also note that $B(c)$ is an increasing and continuous function and that $B(1)=1$. Finally, note that $B^{\prime}(c)$ is bounded above by 1 . Indeed,

$$
\frac{d B^{-1}(x)}{d x}=1-H^{\prime}(x)>1 .
$$

Instead of considering our original initial value problem, consider the following sequence of initial value problems. Let $\left\{c_{m}\right\}$ be an increasing sequence of numbers that converge to 1 . Define the initial value problem

$$
\hat{b}_{m}^{\prime}=\phi\left(\hat{b}_{m}, c\right), \hat{b}_{m}\left(c_{m}\right)=c_{m} .
$$

The function $\phi(b, c)$ and also $\partial \phi(b, c) / \partial b$ are continuous for $c \in[0,1)$ and $b \in[0, B(c))$ if $g(c)$ is positive and continuously differentiable. Thus, there exists a unique solution $\hat{b}_{m}(c)$ to that initial value problem in some interval $\left(c_{m}-T, c_{m}\right]$, for some positive number $T$. We note that the solution $\hat{b}_{m}(c) \in[c, B(c))$. Indeed, $B^{\prime}(c)$ is bounded above by 1 , but $\phi(b, c) \rightarrow \infty$ as $b \rightarrow B(c)$. This shows that indeed $\hat{b}_{m}(c)<B(c)$. We conclude that the unique solution $\hat{b}_{m}(c)$ exists in $\left[0, c_{m}\right]$. Then define the monotone continuous function in the interval $[0,1]$ :

$$
b_{m}(c)=\left\{\begin{array}{ll}
\hat{b}_{m}(c) & \text { if } c<c_{m} \\
c & \text { if } c \geq c_{m}
\end{array}\right\} .
$$


Lemma 1: The sequence $b_{m}(c)$ is non-decreasing in $m$ for all $c \in[0,1]$. Also $b_{m}(c) \in[c, B(c))$. Proof: Consider the functions $b_{m}$ and $b_{m+1}$. At the point $c_{m+1}, b_{m}^{\prime}\left(c_{m+1}\right)=1$, whereas the left derivative of $b_{m+1}\left(c_{m+1}\right)$ is zero. Thus, in an open interval to the left of $c_{m+1}, b_{m+1}(c)>b_{m}(c)$. We show next that the continuous functions $b_{m+1}(c)$ and $b_{m}(c)$ do not cross. Assume that for some $c<c_{m+1}, b_{m+1}(c)=b_{m}(c)$. If $c>c_{m}$, then again $b_{m}{ }^{\prime}(c)=1$ and $b_{m+1}{ }^{\prime}(c)=0$. The latter follows from the fact that $b_{m}(c)=c$ in this interval, and then if $b_{m+1}(c)=b_{m}(c)=c$, the numerator in the definition of $\phi(b, c)$ is zero. Now, if $c \leq c_{m}$, then $b_{m+1}\left(c^{\prime}\right)=b_{m}\left(c^{\prime}\right)$ for all $c^{\prime}<c$. Indeed, both $b_{m}$ and $b_{m+1}$ would be the solution to the same new initial value problem defined by (5) with $b\left(c^{\prime}\right)=b_{m}(c)$ for $c^{\prime}=c$. Thus, we conclude that the functions do not cross, and that indeed $b_{m+1}(c) \geq b_{m}(c)$ for all $c$.

To prove the second part of the lemma, we first show that $b_{m}$ does not cross the function $c$. In the interval $\left[c_{m}, 1\right]$, the two functions coincide. As we have shown above, the left derivative of $b_{m}$ at $c_{m}$ is zero, and then $b_{m}$ is above $c$ at the left of $c_{m}$. If at some $c<c_{m}, b_{m}(c)=c$, then once again the derivative of $b_{m}$ at $c$ is zero, and the derivative of $c$ is one. Thus, we conclude that $b_{m}$ does not cross $c$. QED

A consequence of the first part of Lemma 1 is that $b_{m}(c)-b_{m-1}(c) \rightarrow 0$ for all $c \in[0,1]$. Let $b(c)=\lim _{m \rightarrow \infty} b_{m}(c)$. By the second part of Lemma $1, H\left(b_{m}(c)\right)+c>b_{m}(c) \geq c$, so that $b_{m}{ }^{\prime}(c)>0$. Pointwise convergence implies that $b(c)$ is monotone, but does not guarantee the continuity of $b(c)$. The next lemma shows that $b(c)$ is indeed continuous.

Lemma 2: $b(c)$ is continuous. That is, $b_{m}$ converges uniformly on $[0,1]$.

Proof: Assume that $b(c)$ is discontinuous at a point $c^{\prime}<1$. Let $b_{-}=\lim _{c \uparrow c^{\prime}} b(c)$ and $b_{+}=\lim _{c \downarrow c^{\prime}} b(c)$. Also, let $b_{+}-b_{-}=\alpha>0$. Since $b_{m}(c) \rightarrow b(c)$ and $b_{m}(c)$ is a nondecreasing sequence of continuous monotone functions, then $\forall \varepsilon_{1}, \delta_{1}>0, \exists m$ such that $b_{m}\left(c+\delta_{1}\right)-b_{m}\left(c-\delta_{1}\right)>\alpha-\varepsilon_{1}$, in which case $\exists c \in\left(c^{\prime}-\delta_{1}, c^{\prime}+\delta_{1}\right) \quad$ such that 
$b_{m}{ }^{\prime}(c)=\phi\left(b_{m}(c), c\right) \geq\left(\alpha-\varepsilon_{1}\right) / 2 \delta_{1}$. For $\delta_{1}$ small enough (consider only $\delta_{1}$ smaller than $\left(1-c^{\prime}\right) / 3$ ), the right-hand side is a as large as desired. That is, $\forall \varepsilon_{1}, \delta_{1}>0, \exists m$, $c \in\left(c^{\prime}-\delta_{1}, c^{\prime}+\delta_{1}\right)$ such that $\phi\left(b_{m}(c), c\right)$ is as large as desired. Or equivalently, $b_{m}(c)$ is as close to $B(c)$ as desired and $\left[H\left(b_{m}(c)\right)-\left(b_{m}(c)-c\right)\right]$ is as close to zero as desired. That shows that, $\forall \varepsilon>0, \exists m, c, \delta_{1}$ such that

$$
\phi\left(b_{m}(c), c\right)>\frac{(n-1) \cdot H\left(b_{m}(c)\right)}{H(c) \cdot \varepsilon},
$$

and also $\varepsilon>B(c)-b_{m}(c)$.

Let $\delta_{2}=\left(1-c^{\prime}\right) / 3$. Since $H(c)$ is non-increasing, the above inequality implies

$$
\phi\left(b_{m}(c), c\right)>\frac{(n-1) \cdot H\left(b_{m}\left(c^{\prime}+\delta_{1}+\delta_{2}\right)\right)}{H\left(c^{\prime}\right) \cdot \varepsilon} \equiv \Delta(\varepsilon) .
$$

For $\varepsilon$ small enough, that expression is larger than one, and thus larger than $B^{\prime}(c)$. (Recall that $B^{\prime}(c)$ is bounded above by one.) In other words, for values $c^{\prime \prime}>c, B(c)-b_{m}(c)$ does not become larger. Thus, we can conclude that the inequality above holds for any $c^{\prime \prime}$ in the interval $\left(c, c^{\prime}+\delta_{1}+\delta_{2}\right)$. Take $\varepsilon$ so that $\Delta(\varepsilon) \cdot \delta_{2}>\varepsilon+2 \delta_{2}$, and since $\delta_{2}>\delta_{1}, \varepsilon>B(c)-b_{m}(c)$. and $B^{\prime}\left(c^{\prime}\right)<1$, we conclude that $b_{m}(c)>B(c)$. This contradicts Lemma 1 and then shows that indeed $b(c)$ is continuous at $c<1$. For $c=1$, continuity is a straightforward consequence of our construction of the sequence of functions $b_{m}(c)$. QED

We can now show that the limit, $b(c)$, is a solution to the initial value problem (5) and is strictly monotone. To that end, we first show that $b_{m}{ }^{\prime}$ converges uniformly in $[0,1)$.

Lemma 3: $b_{m}{ }^{\prime}$ converges uniformly in $[0,1)$.

Proof: First notice that $b_{m}{ }^{\prime}$ is well defined except at $c_{m}$. Now, for any point $c$ in [0,1), let M be such that $c<c_{M}$. For all $m>\mathrm{M}, b_{m}{ }^{\prime}(c)=\phi\left(b_{m}(c), c\right)$. According to Lemma $1, b_{m}(c)$ is nondecreasing in $m, b_{m}(c) \geq c$, and also we have that $\phi(b, c)$ is monotone increasing in $b \geq c$. Thus, $b_{m}{ }^{\prime}(c)$ is increasing in $m$ and bounded above by $\phi(b(c), c)$. From Lemma 1, we also know 
that $b(c) \leq B(c)$, but we have to show that $b(c)<B(c)$ in order to guarantee that $\phi(b(c), c)$ (the upper bound) is finite. To this end, note that if $b(c)=B(c)$ then for $m$ large enough $b_{m}{ }^{\prime}(c)=\phi\left(b_{m}(c), c\right)$ is arbitrarily large and at the same time $b_{m}(c)$ arbitrarily close to $B(c)$. Since $B^{\prime}(c)$ is bounded above by 1 , we would have that for some $m, b_{m}$ would cross $B$, which would contradict Lemma 1.

Thus, we can guarantee that $b_{m}{ }^{\prime}(c)$ converges. Let $b^{\prime}(c)$ denote that limit, and assume that it is not continuous. That is, for some c, say $c=1-a$, for some $a \in(0,1]$, there exists $\varepsilon$ such that

$$
\forall \delta, \exists c^{\prime} \text { s.t. }\left|c^{\prime}-c\right|<\delta \text { and }\left|b^{\prime}\left(c^{\prime}\right)-b^{\prime}(c)\right|>\varepsilon .
$$

Then, fix $\delta<a$ so that

i) $\forall c^{\prime \prime}$ s.t. $\left|c^{\prime \prime}-c\right|<\delta,\left|\phi\left(b\left(c^{\prime \prime}\right), c^{\prime \prime}\right)-\phi(b(c), c)\right|<\varepsilon / 5$

and consider the corresponding $c^{\prime}$. We know that $\delta$ exists since $\phi$ is continuous (and so uniformly continuous) in $[0,1-a]$. Next, fix $\mathrm{M}$ such that for all $\mathrm{m}>\mathrm{M}$,

ii) $\left|b_{m}{ }^{\prime}(c)-b^{\prime}(c)\right|<\varepsilon / 5$,

iii) $\left|b_{m}{ }^{\prime}\left(c^{\prime}\right)-b^{\prime}\left(c^{\prime}\right)\right|<\varepsilon / 5$,

iv) $\forall c^{\prime \prime},\left|\phi\left(b_{m}\left(c^{\prime \prime}\right), c^{\prime \prime}\right)-\phi\left(b\left(c^{\prime \prime}\right), c^{\prime \prime}\right)\right|<\varepsilon / 5$.

We know that $\mathrm{M}$ exists since $b_{m}{ }^{\prime}$ converges point-wise to $b^{\prime}$-(ii) and (iii)-, $b_{m}$ converges uniformly to $b$, and again $\phi$ is uniformly continuous -(iv)-. Now,

$$
\left|b^{\prime}\left(c^{\prime}\right)-b^{\prime}(c)\right| \leq\left|b^{\prime}\left(c^{\prime}\right)-b_{m}{ }^{\prime}\left(c^{\prime}\right)\right|+\left|b^{\prime}\left(c^{\prime}\right)-b_{m}{ }^{\prime}(c)\right|+\left|b_{m}{ }^{\prime}\left(c^{\prime}\right)-b_{m}{ }^{\prime}(c)\right|,
$$

And

$$
\begin{aligned}
& \left|b_{m}{ }^{\prime}\left(c^{\prime}\right)-b_{m}{ }^{\prime}(c)\right|=\left|\phi\left(b_{m}\left(c^{\prime}\right), c^{\prime}\right)-\phi\left(b_{m}(c), c\right)\right| \leq \\
& \left|\phi\left(b_{m}\left(c^{\prime}\right), c^{\prime}\right)-\phi\left(b\left(c^{\prime}\right), c^{\prime}\right)\right|+\left|\phi\left(b_{m}(c), c\right)-\phi(b(c), c)\right|+\left|\phi\left(b\left(c^{\prime}\right), c^{\prime}\right)-\phi(b(c), c)\right| .
\end{aligned}
$$

Thus, from (i), (ii), (iii), and (iv), we conclude that $\left|b^{\prime}\left(c^{\prime}\right)-b^{\prime}(c)\right|<\varepsilon$, adn this contradiction proves that $b^{\prime}(c)$ is continuous. That proves the lemma. QED

We are now ready to show that $b(c)$ is a solution to (5). First, note that $b_{m}(1)=1$ for all $m$, so that indeed the initial condition is satisfied, $b(1)=1$. Now, take a sequence of initial values 
problems defined by (5) and the initial condition $b_{m}\left(c_{m}\right)=c_{m}$. Note that $b(c)$ is the solution to each of these initial value problems in the corresponding intervals $\left[0, c_{m}\right]$. Indeed,

$\lim _{\varepsilon \rightarrow 0} \frac{b(c)-b(c-\varepsilon)}{\varepsilon}=\lim _{\varepsilon \rightarrow 0} \lim _{m \rightarrow \infty} \frac{b_{m}(c)-b_{m}(c-\varepsilon)}{\varepsilon}=$

$\lim _{m \rightarrow \infty} \lim _{\varepsilon \rightarrow 0} \frac{b_{m}(c)-b_{m}(c-\varepsilon)}{\varepsilon}=\lim _{m \rightarrow \infty} \phi\left(b_{m}(c), c\right)=\phi(b(c), c)$,

Where the second equality follows from uniform convergence of $b_{m}{ }^{\prime}(c)$ and the fourth from the fact that $\phi(b(c), c)$ is continuous in the interval $\left[0, c_{m}\right]$.

Now, $\left\{c_{m}, b\left(c_{m}\right)\right\} \rightarrow\{1,1\}$, and thus $b(c)$ is indeed the solution to our initial value problem.

Second, we need to show that $b(c)$ is strictly monotone. Assume this is not true, so that at some point $c<1$ we have that $b^{\prime}(c)=0$. That can only happen when $b(c)=c$, in which case the function $b(c)$ would cross the $45^{\circ}$ line at $c$, so that for some $c^{\prime}>c, b\left(c^{\prime}\right)<c^{\prime}$. This contradicts Lemma 1 . Thus, $b(c)$ is strictly monotone. QED 